\title{
A NOTE ON A MONOTONICITY PROPERTY OF BESSEL FUNCTIONS
}

\author{
STAMATIS KOUMANDOS \\ Department of Pure Mathematics \\ The University of Adelaide \\ Adelaide 5005, SOUTH AUSTRALIA \\ Current Address: \\ Department of Mathematics and Statistics \\ University of Cyprus \\ P.O. Box 537 \\ 1678 Nicosia, CYPRUS
}

(Received September 18, 1995)

ABSTRACT. A theorem of Lorch, Muldoon and Szegö states that the sequence

$$
\left\{\int_{J_{a, k}}^{J_{a, k+1}} t^{-\alpha}\left|J_{\alpha}(t)\right| d t\right\}_{k=1}^{\infty}
$$

is decreasing for $\alpha>-1 / 2$, where $J_{\alpha}(t)$ the Bessel function of the first kind order $\alpha$ and $j_{\alpha, k}$ its $k$ th positive root. This monotonicity property implies Szegö's inequality

$$
\int_{0}^{x} t^{-\alpha} J_{\alpha}(t) d t \geq 0
$$

when $\alpha \geq \alpha^{\prime}$ and $\alpha^{\prime}$ is the unique solution of $\int_{0}^{\int_{a, 2}} t^{-\alpha} J_{\alpha}(t) d t=0$

We give a new and simpler proof of these classical results by expressing the above Bessel function integral as an integral involving elementary functions.

KEY WORDS AND PHRASES: Bessel functions, positive integral of Beśsel functions, monotonicity property of Bessel functions.

1991 AMS SUBJECT CLASSIFICATION CODES: 33C10, 33C45.

\section{INTRODUCTION}

Let $J_{\alpha}(t)$ be the Bessel function of the first kind and order $\alpha, j_{\alpha, 1}, j_{\alpha, 2}, \ldots$ its positive roots in increasing order and $j_{\alpha, 0}=0$

In [1] Lorch, Muldoon and Szegö derived, among other things, the following

THEOREM 1. For $\alpha>-\frac{1}{2}$ the sequence of areas

$$
\left\{\int_{J_{a, k}}^{J_{a, k+1}} t^{-\alpha}\left|J_{\alpha}(t)\right| d t\right\}_{k=1}^{\infty}
$$

is decreasing.

As it is shown in [1], this theorem is a special case of a more general result concerning cylinder functions and its proof is based on an application of a Sturm-type oscillation theorem (formulated by 
Watson [2, p. 518], sharpened and applied in greater detail by Makai [3]) to a certain linear differential equation of second order.

In addition, as the authors pointed out in [1], Theorem 1 gives another proof of a classical inequality of Szegö contained in the Notes which he appended to a posthumous paper of Feldheim [4] in the course of preparing it for publication.

Szegö proved in [4] that

$$
\int_{0}^{\jmath^{\prime, 2 k}} t^{-\alpha^{\prime}} J_{\alpha^{\prime}}(t) d t>0, \quad k=2,3, \ldots
$$

where $\alpha^{\prime}$ is the unique root of the transcendental equation

$$
\int_{0}^{\jmath_{a, 2}} t^{-\alpha} J_{\alpha}(t) d t=0
$$

whose numerical value is $\alpha^{\prime}=-0.26938 \ldots$.

As indicated by Szegö, (1.2) in combination with an application of the Sonine integral (see [4, p. $279]$ or [2, p. 373]), yield the inequality

$$
\int_{0}^{x} t^{-\alpha} J_{\alpha}(t) d t>0 \text { for all } x>0, \text { when } \alpha>\alpha^{\prime} .
$$

Szegö's proof of (1.2) is rather intricate as it relies on various properties of Bessel functions as well as of certain identities involving the Lommel functions.

It should be noted that inequality (1.4) suggests a much stronger inequality involving ultraspherical polynomials which was recently established in [5].

Over the years, generalizations of (1.4) have been proved by several authors In particular, the inequality

$$
\int_{0}^{x} t^{-\beta} J_{\alpha}(t) d t>0, \quad x>0, \quad \beta<\alpha+1,
$$

was proved by Makai [6] for $-\frac{1}{2}<\alpha<\frac{1}{2}$ and $\beta(\alpha) \leq \beta<\alpha+1$, where

$$
\int_{0}^{j_{a, 2}} t^{-\beta(\alpha)} J_{\alpha}(t) d t=0
$$

Askey and Steinig [7] proved (1.5) for $-1<\alpha<-\frac{1}{2}$ for the same range of $\beta$. For $\alpha=-\frac{1}{2}$, (1.5) turns out to be a classical inequality for cosine integrals. When $\alpha \geq \frac{1}{2}$, (1.5) holds for $-\frac{1}{2} \leq \beta<\alpha+1$ and this follows from a work of Gasper [8], in which an explicit expression of the integral in question as a sum of squares of the Bessel functions with positive coefficients is proved. See also [9] for some more recent results on positive integrals of Bessel functions.

The purpose of this note is to show that Theorem 1 can be established in a simpler manner than that in [1] and hence to give a new and more straightforward proof of Szegö's inequalities (1.2) and (1.4)

We proceed by observing that Theorem 1 is equivalent to

THEOREM 2. For $\alpha>-\frac{1}{2}$, we define

$$
f(x, \alpha)=\int_{0}^{x} t^{-\alpha} J_{\alpha}(t) d t, \quad x>0
$$

Then, the local minima of $f(x, \alpha)$, as a function of $x$, form an increasing sequence, i.e.

$$
f\left(j_{\alpha, 2 \ell}, \alpha\right)<f\left(j_{\alpha, 2 \ell+2}, \alpha\right), \quad \ell=1,2, \ldots
$$

and its local maxima form a decreasing sequence, i.e. 


$$
f\left(J_{\alpha, 2 \ell-1}, \alpha\right)>f\left(j_{\alpha, 2 \ell+1}, \alpha\right), \quad \ell=1,2, \ldots .
$$

It is well known that the graph of $y=J_{\alpha}(t),(\alpha>-1)$ consists of waves alternately above and below the axis of $t$, whose areas form a steadily decreasing sequence, $t$ being positive. This classical result was proved originally by Cooke in [10] and [11]. Cooke's proof is rather complicated as it depends on some delicate estimates involving the Lommel functions and several properties of Bessel functions In [3], Makai proved this result for $|\alpha|>\frac{1}{2}$ in a simpler way using a differential equation approach of Sturm-Liouville type. A particularly simple proof of Cooke's Theorem has been devised by Steinig in [12].

Since the sequence

$$
\left\{\int_{J_{a, k}}^{J_{a, k+1}}\left|J_{\alpha}(t)\right| d t\right\}_{k=1}^{\infty}
$$

is steadily decreasing and for $\alpha>0, t^{-\alpha}$ is a positive decreasing function of $t$, we have

$$
\begin{aligned}
\int_{J_{\alpha, k}}^{J_{a, k+1}} t^{-\alpha}\left|J_{\alpha}(t)\right| d t \geq \int_{j_{a, k}}^{J_{a, k+1}}\left(j_{\alpha, k+1}\right)^{-\alpha}\left|J_{\alpha}(t)\right| d t \\
\quad>\int_{J_{\alpha, k+1}}^{J_{a, k+2}}\left(j_{\alpha, k+1}\right)^{-\alpha}\left|J_{\alpha}(t)\right| d t \geq \int_{J_{\alpha, k+1}}^{J_{a, k+2}} t^{-\alpha}\left|J_{\alpha}(t)\right| d t,
\end{aligned}
$$

which establishes Theorem 1 for all $\alpha>0$. It is clear that the case $\alpha=0$ reduces to Cooke's result as well.

In the next section we give a simple proof of Theorem 2 for the range $-\frac{1}{2}<\alpha<0$ This is, of course, the interesting case as the critical value $\alpha^{\prime}$ for which the Szegö's inequalities (1.2) and (1 4) are valid, is contained in this interval.

2. PROOF OF THEOREM 2 FOR $-\frac{1}{2}<\alpha<0$

For this proof we need the following elementary lemma.

LEMMA. Let $0<\mu<1$ and

$$
g(t)=\frac{\sin \pi t}{t\left(1-t^{2}\right)^{\mu}} \quad \text { for } \quad 0<t<1 .
$$

Then we have $g^{\prime}(t)<0$ for $t \in(0,1)$. Moreover, $g^{\prime \prime}(t)<0$ for $t \in(0,1)$, when $\frac{1}{2}<\mu<1$

PROOF. We observe that

$$
\left(1-t^{2}\right)^{\mu+1} g^{\prime}(t)=\frac{1-t^{2}}{t^{2}}(\pi t \cos \pi t-\sin \pi t)+2 \mu \sin \pi t .
$$

To prove the negativity of $g^{\prime}(t)$, it suffices to show that

$$
\frac{1-t^{2}}{t^{2}}(\pi t \cos \pi t-\sin \pi t)+2 \sin \pi t<0,
$$

or equivalently

$$
\pi t \operatorname{ctg}(\pi t)<\frac{1-3 t^{2}}{1-t^{2}}
$$

Now taking into account the familiar formula

$$
\pi t \operatorname{ctg}(\pi t)=1+2 t^{2} \sum_{k=1}^{\infty} \frac{1}{t^{2}-k^{2}}
$$

we see that (2.1) is equivalent to 


$$
\sum_{k=2}^{\infty} \frac{1}{k^{2}-t^{2}}>0
$$

which is apparently true for $0<t<1$. Hence, $g^{\prime}(t)<0$, for $0<t<1$.

Suppose that $\frac{1}{2}<\mu<1$. A routine calculation shows that the negativity of $g^{\prime \prime}(t)$, for $0<t<1$, follows from the inequality

$$
\pi^{2}\left(1-t^{2}\right)^{2}-2 \mu\left[1+(2 \mu+1) t^{2}\right]+2 \frac{\left(1-t^{2}\right)^{2}}{t^{2}}(\pi t \operatorname{ctg} \pi t-1)-4 \mu\left(1-t^{2}\right)(\pi t \operatorname{ctg} \pi t-1)>0 .
$$

In view of (2.2), this is equivalent to

$$
\pi^{2} t^{4}+\left(6 \mu+4-4 \mu^{2}-2 \pi^{2}\right) t^{2}+\pi^{2}-2 \mu-4-4\left(1-t^{2}\right)\left(1-(2 \mu+1) t^{2}\right) \sum_{k=2}^{\infty} \frac{1}{k^{2}-t^{2}}>0 .
$$

Since

$$
\frac{\pi^{2}}{6}-1<\sum_{k=2}^{\infty} \frac{1}{k^{2}-t^{2}}<\frac{3}{4}, \quad 0<t<1
$$

inequality (2.3) follows easily by an elementary computation.

The proof of the lemma is complete.

Now, in order to prove Theorem 2 we observe that the integral in (1.7) that defines the function $f(x, \alpha)$ coincides with an integral of certain elementary functions.

In fact, by Poisson's integral (cf. [2, p. 48])

$$
J_{\alpha}(z)=\frac{2\left(\frac{1}{2} z\right)^{\alpha}}{\Gamma\left(\alpha+\frac{1}{2}\right) \Gamma\left(\frac{1}{2}\right)} \int_{0}^{1}\left(1-t^{2}\right)^{\alpha-\frac{1}{2}} \cos (z t) d t, \quad \text { for } \quad \alpha>-\frac{1}{2},
$$

it follows easily that

$$
\int_{0}^{x} t^{-\alpha} J_{\alpha}(t) d t=\frac{2^{1-\alpha}}{\Gamma\left(\frac{1}{2}\right) \Gamma\left(\alpha+\frac{1}{2}\right)} \int_{0}^{1} \frac{\sin (x t)}{t} \frac{d t}{\left(1-t^{2}\right)^{\frac{1}{2}-\alpha}}
$$

for $x>0$ and $\alpha>-\frac{1}{2}$.

Since the zeros of $J_{\alpha}(t)$ are increasing with $\alpha[2$, p. 508] and

$$
J_{-\frac{1}{2}}(t)=\left(\frac{2}{\pi t}\right)^{\frac{1}{2}} \cos t, \quad J_{\frac{1}{2}}(t)=\left(\frac{2}{\pi t}\right)^{\frac{1}{2}} \sin t,
$$

we have for $-\frac{1}{2}<\alpha<\frac{1}{2}$

$$
\left(\nu-\frac{1}{2}\right) \pi<j_{\alpha, \nu}<\nu \pi, \quad \nu=1,2, \ldots .
$$

In addition, Szegö showed in [13] that, for $-\frac{1}{2}<\alpha<\frac{1}{2}$,

$$
j_{\alpha, \nu}-j_{\alpha, \nu-1}<\pi, \quad \nu=1,2, \ldots .
$$

Combining this with (2 5) we get

$$
j_{\alpha, \nu+2}-j_{\alpha, \nu}<2 \pi<j_{\alpha, \nu+3}-j_{\alpha, \nu}, \quad \nu=1,2, \ldots
$$

when $-\frac{1}{2}<\alpha<\frac{1}{2}$

Let

$$
\phi(x, \alpha)=\int_{0}^{1} \frac{\sin (x t)}{t} \frac{d t}{\left(1-t^{2}\right)^{\frac{1}{2}-\alpha}}
$$


Taking into consideration (2.4) and (2.6) we see that in order to prove (1.8) it suffices to show that

$$
\phi(x, \alpha)-\phi(x-2 \pi, \alpha)>0, \quad \text { for } 2 \ell \pi+\frac{3 \pi}{2}<x<2 \ell \pi+2 \pi, \quad \ell=1,2, \ldots .
$$

Similarly, (1.9) can be obtained by showing

$$
\phi(x, \alpha)-\phi(x-2 \pi, \alpha)<0, \quad \text { for } \quad 2 \ell \pi+\frac{\pi}{2}<x<2 \ell \pi+\pi, \quad \ell=1,2, \ldots .
$$

It is evident that (2.7) is equivalent to

$$
\int_{0}^{1} \cos (y t) \frac{\sin (\pi t)}{t\left(1-t^{2}\right)^{\frac{1}{2}-\alpha}} d t>0 \text { for } 2 \ell \pi+\frac{\pi}{2}<y<2 \ell \pi+\pi, \quad \ell=1,2, \ldots
$$

which, in turn, is equivalent to

$$
\int_{0}^{y} K_{y}(t) \cos t d t>0, \quad 2 \ell \pi+\frac{\pi}{2}<y<2 \ell \pi+\pi, \quad \ell=1,2, \ldots
$$

where

$$
K_{y}(t)=\frac{\sin \left(\frac{\pi t}{y}\right)}{t\left(y^{2}-t^{2}\right)^{\frac{1}{2}-\alpha}}, \quad 0<t<y
$$

We have

$$
\begin{aligned}
\int_{0}^{y} K_{y}(t) \cos t d t & =\sum_{j=1}^{\ell} \int_{0}^{2 \pi} K_{y}((2 j-2) \pi+t) \cos t d t+\int_{0}^{y-2 \ell \pi} K_{y}(2 \ell \pi+t) \cos t d t \\
& =\int_{0}^{\frac{\pi}{2}} T_{y}^{\ell}(t) \cos t d t-\int_{(2 \ell+1) \pi-y}^{\frac{\pi}{2}} K_{y}((2 \ell+1) \pi-t) \cos t d t
\end{aligned}
$$

where

$$
\begin{aligned}
& T_{y}^{\ell}(t)=\sum_{j=1}^{\ell}\left\{K_{y}((2 j-2) \pi+t)-K_{y}((2 j-1) \pi-t)\right. \\
&\left.-K_{y}((2 j-1) \pi+t)+K_{y}(2 j \pi-t)\right\}+K_{y}(2 \ell \pi+t) .
\end{aligned}
$$

We observe that the function

$$
\Delta_{y}^{\ell}(t)=T_{y}^{\ell}(t)-K_{y}((2 \ell+1) \pi-t)
$$

is decreasing for $(2 \ell+1) \pi-y<t<\frac{\pi}{2}$, since it has the form

$$
\Delta_{y}^{\ell}(t)=Q_{y}^{\ell}(t)-Q_{y}^{\ell}(\pi-t),
$$

where

$$
Q_{y}^{\ell}(t)=K_{y}(t)+\sum_{j=1}^{\ell}\left\{K_{y}(2 j \pi+t)+K_{y}(2 j \pi-t)\right\} .
$$

By the lemma of this section it follows that $Q_{y}^{\ell}(t)$ is a decreasing function of $t$, therefore

$$
\Delta_{y}^{\ell}(t)>\Delta_{y}^{\ell}\left(\frac{\pi}{2}\right)=0, \quad(2 \ell+1) \pi-y<t<\frac{\pi}{2}, \quad \ell=1,2, \ldots .
$$

From this it follows that

$$
T_{y}^{\ell}(t)>0, \quad 0<t<\frac{\pi}{2}, \quad \ell=1,2, \ldots
$$


Finally, by (2.10), (2.11) and (2.12) we get

$$
\int_{0}^{y} K_{y}(t) \cos t d t>\int_{(2 \ell+1) \pi-y}^{\frac{\pi}{2}} \Delta_{y}^{\ell}(t) \cos t d t>0,
$$

which gives (2.9). By a similar argument we establish (2.8) and complete the proof of Theorem 2.

\section{REFERENCES}

[1] LORCH, L., MULDOON, M.E. and SZEGÖ, P, Some monotonicity properties of Bessel functions, SIAM J. Math. Anal. 4 (1973), 385-392.

[2] WATSON, G.N., A Treatise on the Theory of Bessel Functions, 2nd ed., Cambridge University Press, 1944.

[3] MAKAI, E., On a monotonic property of certain Sturm-Liouville functions, Acta Math. Acad. Scl. Hungar. 3 (1952), 165-172.

[4] FELDHEIM, E., On the positivity of certain sums of ultraspherical polynomials, J. Analyse Math. 11 (1963), 275-284 (edited with additional notes by G. Szegö), also in G. Szego collected papers vol. 3, Birkhäuser Boston, 1982, 821-830.

[5] BROWN, G., KOUMANDOS, S. and WANG, K-Y., Positivity of basic sums of ultraspherical polynomials, submitted.

[6] MAKAI, E., An integral inequality satisfied by Bessel functions, Acta Math. Acad. Sci. Hungar. 25 (1974), 387-390.

[7] ASKEY, R. and STEINIG, J., Some positive trigonometric sums, Trans. Amer. Math. Soc. 187 (1974), 295-307.

[8] GASPER, G., Positive integrals of Bessel functions, SIAM J. Math. Anal. 6 (1975), 868-881.

[9] MISIEWICZ, J.K. and RICHARDS, D.S.P., Positivity of integrals of Bessel functions, SIAM J. Math. Anal. 25-2 (1994), 596-601.

[10] COOKE, R.G., Gibbs' phenomenon in Fourier-Bessel series and integrals, Proc. London Math. Soc. 27 (1927), 171-192.

[11] COOKE, R.G, A monotonic property of Bessel functions, J. London Math. Soc. 12 (1937), 180185.

[12] STEINIG, J., On a monotonicity property of Bessel functions, Math. Z. 122 (1971), 363-365

[13] SZEGÖ, G., Inequalities for the zeros of Legendre polynomials and related functions, Trans. Amer. Math. Soc. 39 (1936), 1-17, also in G. Szego collected papers vol. 2, Birkhauser Boston, 1982, 593-610. 


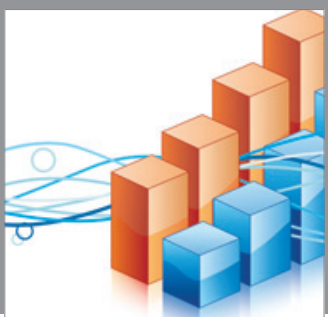

Advances in

Operations Research

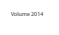

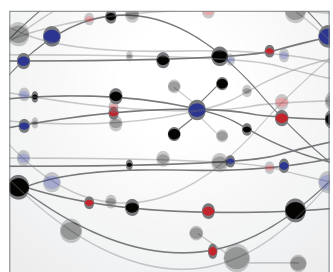

\section{The Scientific} World Journal
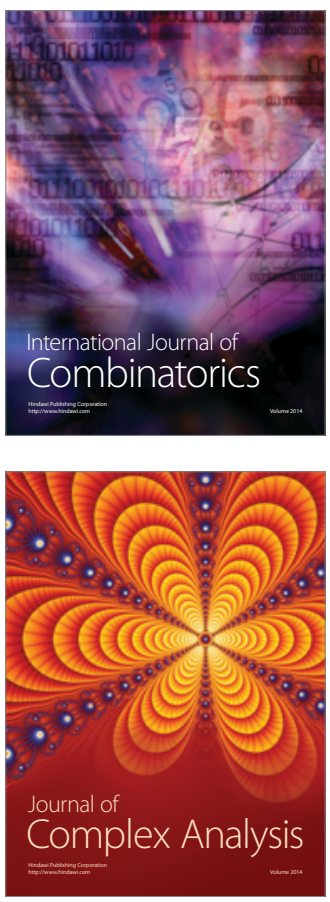

International Journal of

Mathematics and

Mathematical

Sciences
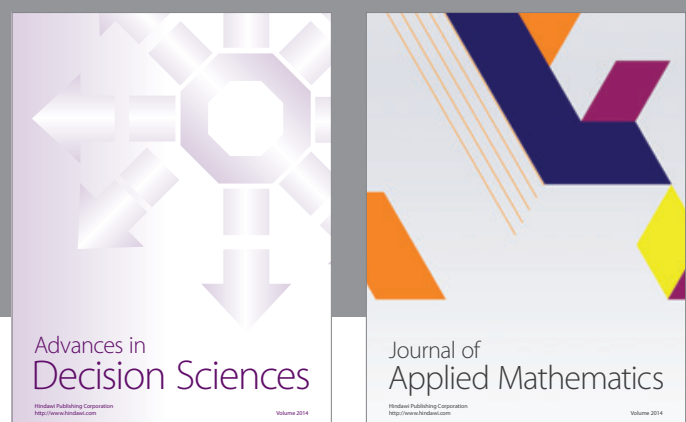

Journal of

Applied Mathematics
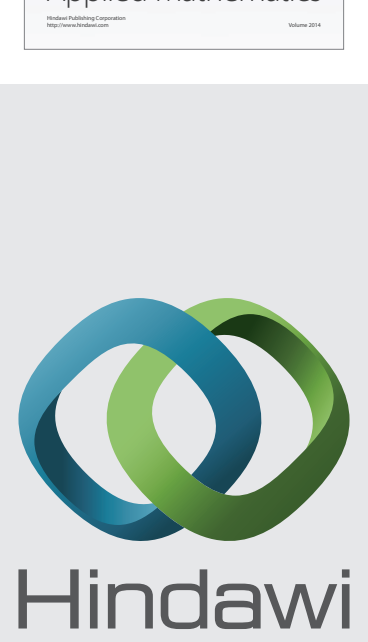

Submit your manuscripts at http://www.hindawi.com
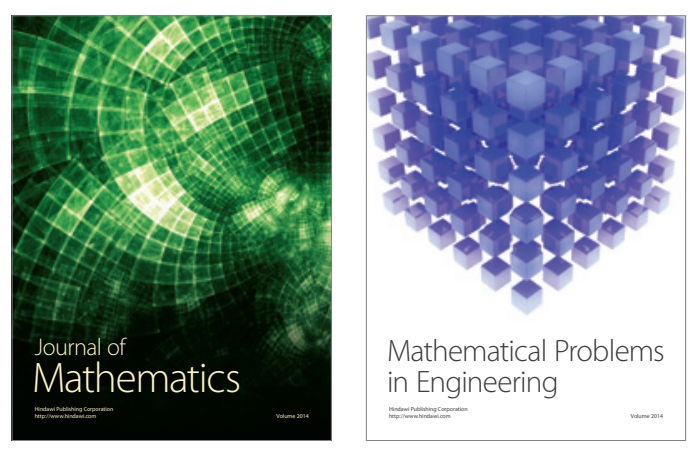

Mathematical Problems in Engineering
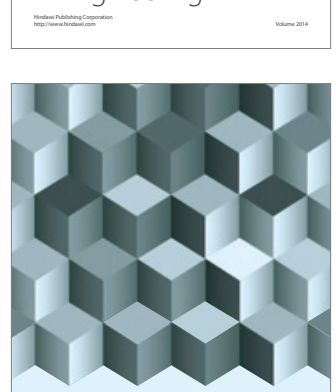

Journal of

Function Spaces
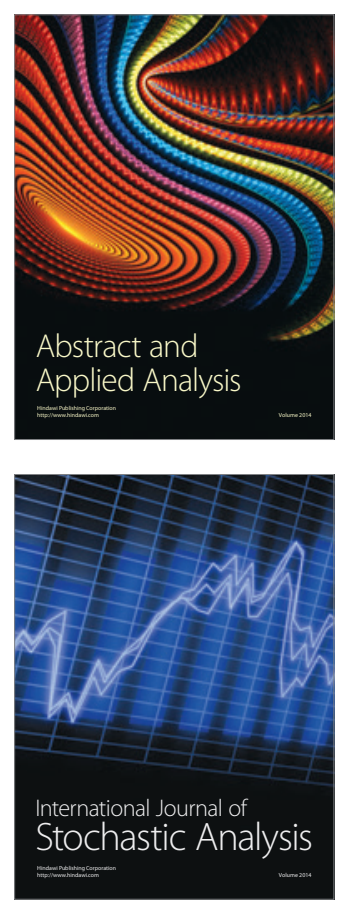

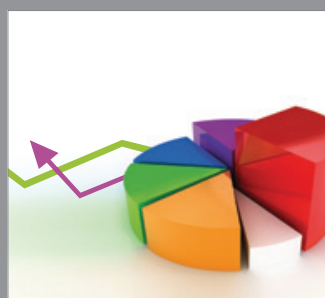

ournal of

Probability and Statistics

Promensencen
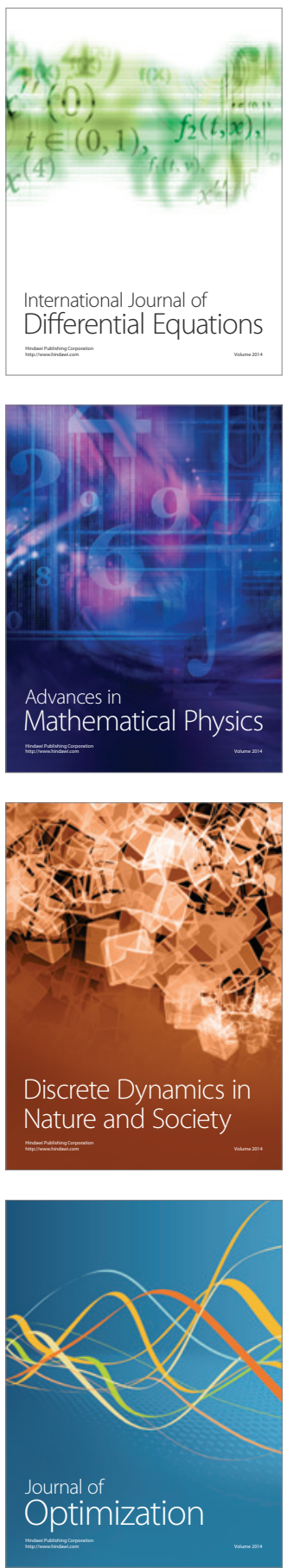